\title{
Oxygen Vacancy Effects of Two-Dimensional Electron Gas in $\mathrm{SrTiO}_{3} / \mathrm{KNbO}_{3}$ Hetero Structure
}

\author{
Woo-Sung Choi ${ }^{1,2}$, Min-Gyu Kang11, Young-Ho Do', Woo-Suk Jung1, Byeong-Kwon Ju², Seok-Jin Yoon², \\ Kwang-Soo Yoo ${ }^{4}$, and Chong-Yun Kang1,5,+
}

\begin{abstract}
The discovery of a two-dimensional electron gas (2DEG) in $\mathrm{LaAlO}_{3}(\mathrm{LAO}) / \mathrm{SrTiO}_{3}(\mathrm{STO})$ heterostructure has stimulated intense research activity. We suggest a new structure model based on $\mathrm{KNbO}_{3}(\mathrm{KNO})$ material. The $\mathrm{KNO}$ thin films were grown on $\mathrm{TiO}_{2-}$ terminated STO substrates as a p-type structure $\left(\mathrm{NbO}_{2} / \mathrm{KO} / \mathrm{TiO}_{2}\right)$ to form a two-dimensional hole gas (2DHG). The STO thin films were grown on $\mathrm{KNO} / \mathrm{TiO}_{2}$-terminated $\mathrm{STO}$ substrates as an n-type structure to form a 2DEG. Oxygen pressure during the deposition of the KNO and STO thin films was changed so as to determine the effect of oxygen vacancies on 2DEGs. Our results showed conducting behavior in the n-type structure and insulating properties in the p-type structure. When both the KNO and STO thin films were deposited on a $\mathrm{TiO}_{2}$-terminated STO substrate at a low oxygen pressure, the conductivity was found to be higher than that at higher oxygen pressures. Furthermore, the heterostructure formed at various oxygen pressures resulted in structures with different current values. An $\mathrm{STO} / \mathrm{KNO}$ heterostructure was also grown on the STO substrate, without using the buffered oxide etchant (BOE) treatment, so as to confirm the effects of the polar catastrophe mechanism. An STO/KNO heterostructure grown on an STO substrate without BOE treatment did not exhibit conductivity. Therefore, we expect that the mechanics of 2DEGs in the STO/KNO heterostructures are governed by the oxygen vacancy mechanism and the polar catastrophe mechanism.
\end{abstract}

Keywords : KNO, 2DEG, STO, Oxygen vacancy, Polar catastrophe

\section{INTRODUCTION}

Oxide materials are known to exhibit a variety of interesting physical phenomena (such as symmetry breaking, charge transfer, strain, frustration, and electrostatic coupling) and a variety of physical properties (from insulating to metallic, magnetic, and even superconducting, depending on the cation and the crystal

${ }^{1}$ Electronic Materials Research Center, Korea Institute of Science and Technology, 39-1, Hawolgok-Dong, Sungbuk-Ku, Seoul, 136-791, Republic of Korea

${ }^{2}$ Display and Nanosystem Laboratory, College of Engineering, Korea University, Seoul 136-713, Republic of Korea

${ }^{3}$ Research Planning \& Coordination Division, Korea Institute of Science and Technology, 39-1, Hawolgok-Dong, Sungbuk-Ku, Seoul 136-791, Republic of Korea

${ }^{4}$ Materials Science and Engineering, University of Seoul, 90, Jeonnongdong, Dongdaemun-gu, Seoul 130-743, Republic of Korea

${ }_{5}$ KU-KIST Graduate School of Converging Science and Technology,

Korea University, Seoul 136-713, Republic of Korea

+Corresponding author: cykang@kist.re.kr

(Received : May.. 15, 2013, Revise : Jun. 27, 2013, Accepted : Jul. 8, 2013)

This is an Open Access article distributed under the terms of the Creative Commons Attribution Non-Commercial License(http://creativecommons.org/licenses/bync/3.0)which permits unrestricted non-commercial use, distribution, and reproduction in any medium, provided the original work is properly cited. structure) [1-4]. Recently, an interesting physical phenomenon has been found at the interface between two oxide materials called the two-dimensional electron gas (2DEG). The interfaces defined by the 2DEG exhibit charge transport behavior so as to equalize the electronic chemical potentials of the two materials [5]. The conducting behavior between the oxide interfaces is useful for creating sensors, two-dimensional conductors, and other electronics applications. Since Ohtomo et al. reported the existence of 2DEG at the heterointerface between two insulating oxides, $\mathrm{LaAlO}_{3}(\mathrm{LAO})$ and $\mathrm{SrTiO}_{3}$ (STO), the origins of the $2 \mathrm{DEG}$ have been investigated to explain the conducting behavior at the heterointerface, such as the polar catastrophe model, oxygen vacancy model, band gap model, and lattice distortion model $[6,7]$. LAO is a polar material that consists of alternately charged atomic layers of $(\mathrm{LaO})^{+}$and $\left(\mathrm{AlO}_{2}\right)^{-}$. STO is a non-polar material that consists of neutral layers of $(\mathrm{SrO})^{0}$ and $\left(\mathrm{TiO}_{2}\right)^{0}$. Half an electron per two-dimensional unit cell passes through the interface, from the LAO to the STO, causing the interfacial $\mathrm{Ti}$ ion in the STO to be in the mixed-valence state $\mathrm{Ti}^{+3.5}$. These half electrons at the $\mathrm{LaO}^{+} / \mathrm{TiO}_{2}^{-0.5}$ interface form the 
2DEG [7-9]. Oxygen vacancies also dope the STO substrate with electrons and cause the interface to conduct $[5,6,10]$. Materials having different band gaps (LAO $\approx$ $5.6 \mathrm{eV}, \mathrm{STO} \approx 3.2 \mathrm{eV}$ ) form a heterojunction, which results in a $2 \mathrm{DEG}$ with a quantum well. The lattice match of two materials affects the conductivity at the heterointerface. The lattice mismatch of LAO/STO is about $3 \%$. The moderate lattice mismatch of $3 \%$ of $\mathrm{SrTiO}_{3}$ allows the epitaxial growth of LAO films on the STO.

In this paper, we present the behavior of a 2DEG at the novel heterointerface between $\mathrm{KNbO}_{3}$ (KNO) and STO. To determine the conductivity of the 2DEG, the interface structure and oxygen vacancies are governed by both the polar catastrophe model and the oxygen vacancy model. According to the principles of the band structure, the $\left(\mathrm{NbO}_{2}\right)^{+} /(\mathrm{SrO})^{0}$ heterointerface in a $\mathrm{KNO} / \mathrm{STO}$ heterostructure is similar to the $(\mathrm{LaO})^{-} /\left(\mathrm{TiO}_{2}\right)^{0}$ heterointerface in a LAO/STO heterostructure $[6,7,11$, 12]. However, because it is difficult to realize $(\mathrm{SrO})^{0}$ terminated surfaces on a single-crystal STO substrate, the $\mathrm{KNO} / \mathrm{STO}$ heterostructure has always used the $(\mathrm{KO})^{-}$ $/\left(\mathrm{TiO}_{2}\right)^{0}$ heterointerface, thus creating a two-dimensional hole gas (2DHG), as shown in Figure 1(a). Therefore, we suggest a novel heterostructure of STO (thin film)/KNO (thin film)/STO (substrate) to create a 2DEG at the $\left(\mathrm{NbO}_{2}\right)^{+} /(\mathrm{SrO})^{0}$ interface between the KNO and STO thin films (Figure 1(b)). The effect of oxygen vacancies and interface structures on the conductivity of the $2 \mathrm{DEG}$ is investigated at the $\mathrm{KNO} / \mathrm{STO}$ interfaces.

\section{EXPERIMENTAL}

The KNO and KNO/STO thin films were grown on TiO2-terminated single crystal STO (001) substrates by pulsed laser deposition (PLD). The $\mathrm{TiO}_{2}$-terminated STO substrates were prepared using buffered oxide etching (BOE) with heat treatment at $900^{\circ} \mathrm{C}$ for $5 \mathrm{~h}$ in oxygen atmosphere [13]. The distance between the target and the substrate, energy density, repetition rate, and substrate temperature were maintained at $5 \mathrm{~cm}, 1.5 \mathrm{~J} / \mathrm{cm}^{2}, 2 \mathrm{~Hz}$, and $700^{\circ} \mathrm{C}$ during the deposition process, respectively. The thickness of the final KNO films was fixed to 8.5 unit cells, which is larger than the critical thickness required to form a 2DEG. The STO films were fixed at $8 \mathrm{~nm}$ in thickness. To control the oxygen vacancies in both films, the oxygen pressure was set to either 1 mTorr or 200 mTorr during the deposition. The oxygen pressures of 200 mTorr and 1 mTorr have been determined to be extreme conditions that are suitable for making films with low and high concentrations of oxygen, respectively. To confirm the presence of polar catastrophe effects, the STO/KNO heterostructure was grown on an STO substrate, without using the BOE treatment. The surface morphologies of the samples were analyzed using atomic force microscopy (AFM, D3100). In-plane I-V characteristics were measured using a probe system (4200-SCS, Keithley).

a)
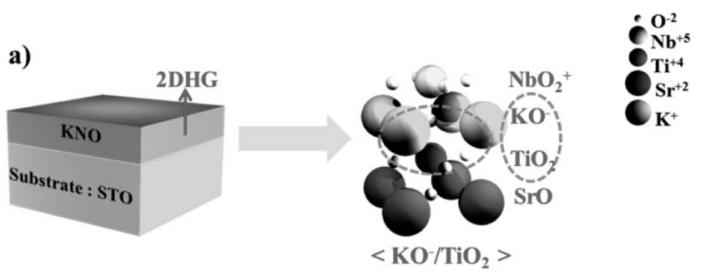

b)

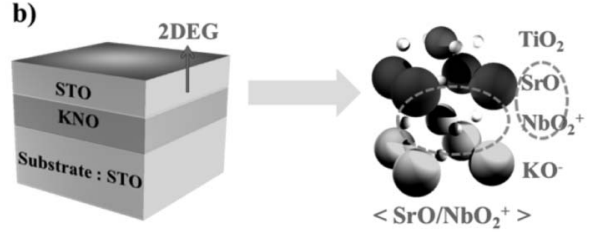

Fig. 1. (a) KNO/STO heterostructure creating $2 \mathrm{DHG}$ and (b) $\mathrm{STO} / \mathrm{KNO} / \mathrm{STO}$ heterostructure creating $2 \mathrm{DEG}$.

\section{RESULTS AND DISCUSSIONS}

Figure 2 (a) shows the in-plane I-V characteristics of the $\mathrm{KNO} / \mathrm{TiO}_{2}$-terminated STO substrate interface that has a $(\mathrm{KO})^{-} /\left(\mathrm{TiO}_{2}\right)^{0}$ heterointerface as a p-type component to create a $2 \mathrm{DHG}$. According to basic materials principles, both 2DEGs and 2DHGs should exhibit conducting behavior. However, the $(\mathrm{KO})^{-} /\left(\mathrm{TiO}_{2}\right)^{0}$ heterointerface has a low current level (acting as an insulator) in spite of the ptype heterointerface creating a $2 \mathrm{DHG}$. According to Ohtomo et al., the mechanism governing conducting behavior in the $2 \mathrm{DEG}$ is explained as electronic reconstruction due to a polar discontinuity at the n-type heterointerface. In the $(\mathrm{KO})^{-} /\left(\mathrm{TiO}_{2}\right)^{0}$ heterointerface, half electron transfers from $\mathrm{TiO}_{2}$ to $\mathrm{KO}$ - due to charge neutrality form $\mathrm{Ti}^{+4.5}$ ions by reconstruction $[6,7]$. However, it is difficult to create charged $\mathrm{Ti}^{+4.5}$ ions because the $\mathrm{Ti}^{+4}$ ion is a more stable, lower energy state [7]. Moreover, the oxygen vacancies in the films disturb both hole generation at the interface and the formation of a 
2DHG.

The in-plane I-V characteristics of the novel heterostructure, namely STO/KNO/STO, are shown in Fig. 2 (b). The new heterointerface designed to create a $2 \mathrm{DEG}$ at the n-type interface of $(\mathrm{SrO})^{0} /\left(\mathrm{NbO}_{2}\right)^{+}$is shown in Fig. 2 (b) [12]. The STO (1 mTorr)/KNO (1 mTorr)/ $/ \mathrm{TiO}_{2}-$ terminated $\mathrm{SrTiO}_{3}$ structure creates a $2 \mathrm{DEG}$ with a high current value of $\approx 10^{-6} \mathrm{~A}$. The AFM images on the right side in Fig. 2 represent the surface morphologies of the fabricated films. The terrace structure is observed in the STO substrate with BOE treatment because the Titerminated surface has flat terraces separated by unit-cell steps. As shown in Fig. 2 (a), the surface morphology of the $\mathrm{KNO} / \mathrm{STO}$ structure of the p-type interface exhibits a terrace structure, which is evidence of KNO epitaxial growth on the $\mathrm{TiO}_{2}$-terminated $\mathrm{STO}$ substrate, whereas terraces are not found in the $\mathrm{STO} / \mathrm{KNO} / \mathrm{STO}$ structure in Fig. 2 (b). It appears that the lattice parameters or film orientation of the STO thin films were changed when the films were deposited on the KNO films [14].
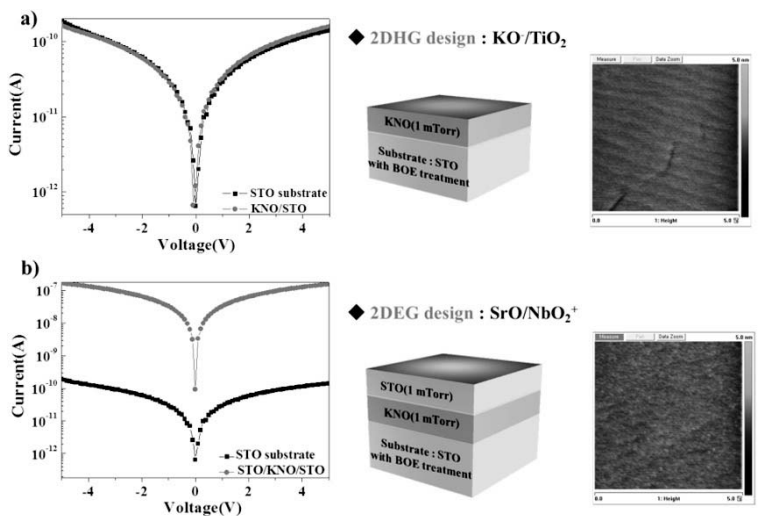

Fig. 2. I-V characteristics and AFM image of (a) KNO/STO heterostructure creating 2DHG (oxygen pressure of 1 mTorr for $\mathrm{KNO}$ ) and (b) $\mathrm{STO} / \mathrm{KNO} / \mathrm{STO}$ heterostructure creating 2DEG (oxygen pressure of $1 \mathrm{mTorr}$ for $\mathrm{KNO}$ and STO).

The STO might exhibit metallic behavior, even if it contains a small number of oxygen vacancies. Therefore, the effects of oxygen vacancy should be considered a component of the 2DEG mechanics [9]. Figure 3 shows the in-plane I-V curve of the STO/KNO/STO structure for different oxygen pressures during deposition. To reduce the effect of oxygen vacancies on the $2 \mathrm{DEG}$ characteristics, $\mathrm{KNO}$ and STO thin films were grown on $\mathrm{TiO}_{2}$-terminated STO substrates at 200 mTorr. The interface between the
KNO and STO thin films grown at 200 mTorr had low current values that indicate the absence of a $2 \mathrm{DEG}$. The KNO (1 mTorr)/STO (200 mTorr) structure and KNO (200 mTorr)/STO (1 mTorr) structure that were deposited on $\mathrm{TiO}_{2}$-terminated STO are observed to have a higher current value than that of the KNO (200 mTorr)/STO (200 mTorr) structure; however, their currents are lower than that of the KNO (1 mTorr)/STO (1 mTorr) structure. This result indicates that the oxygen vacancies in the STO and $\mathrm{KNO}$ thin films contribute to forming the 2DEG. The higher current values of the KNO (200 mTorr)/STO (1 mTorr) structure than that of the KNO (1 mTorr)/STO (200 mTorr) structure is caused by the difference in the thickness of the films. Because the thickness of the KNO film is larger than that of the STO, the KNO thin film contains a larger number of oxygen vacancies than the STO thin films. This result indicates that the lower oxygen pressure of the STO and KNO thin films leads to a higher conductivity at the heterointerface, because a large number of oxygen vacancies are created at lower oxygen pressures and higher thicknesses. Furthermore, the heterointerfaces formed under various oxygen pressures exhibited various current values. As a result, the current level of the 2DEG can easily be controlled by changing the oxygen pressure of the STO and KNO thin films during deposition.

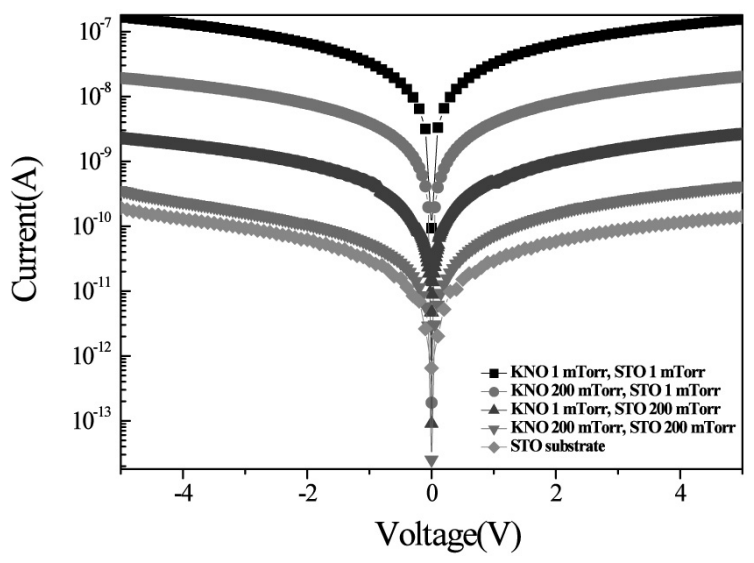

Fig. 3. Transport measurements (the I-V characteristics) of $\mathrm{STO} / \mathrm{KNO} / \mathrm{STO}$ heterostructure for a variety of oxygen pressures during the deposition of the STO and KNO thin films.

As mentioned above, the mechanics of the 2DEG at the $\mathrm{STO} / \mathrm{KNO}$ heterointerface are governed by the effect of the oxygen vacancies, which is the origin of the conducting behavior. In the 2DEG mechanics, the band gap model and 
the lattice distortion model must be considered in certain cases. However, the polar catastrophe model is applicable to the $\mathrm{STO} / \mathrm{KNO}$ heterostructure when considering the mechanics of 2DEGs. To investigate the influence of the polar discontinuity according to the polar catastrophe model, the STO (1 mTorr)/KNO (1 mTorr) thin films were deposited on an STO substrate, without using the BOE treatment. Figure 4 shows the in-plane I-V characteristics of the STO (1 mTorr)/KNO (1 mTorr)/STO substrate, without the BOE treatment. The $\mathrm{STO} / \mathrm{KNO}$ interface grown on the STO substrate without using the BOE treatment exhibits a low current level (acting like an insulator), whereas the STO/KNO interface grown on the $\mathrm{TiO}_{2}$-terminated surface of the STO substrate that forms the n-type interface, namely $(\mathrm{SrO})^{0} /\left(\mathrm{NbO}_{2}\right)^{+}$, between the STO and KNO thin film exhibits a high current value. These results indicate that both the oxygen vacancy model and the polar catastrophe model should be used when analyzing the STO/KNO heterostructure.

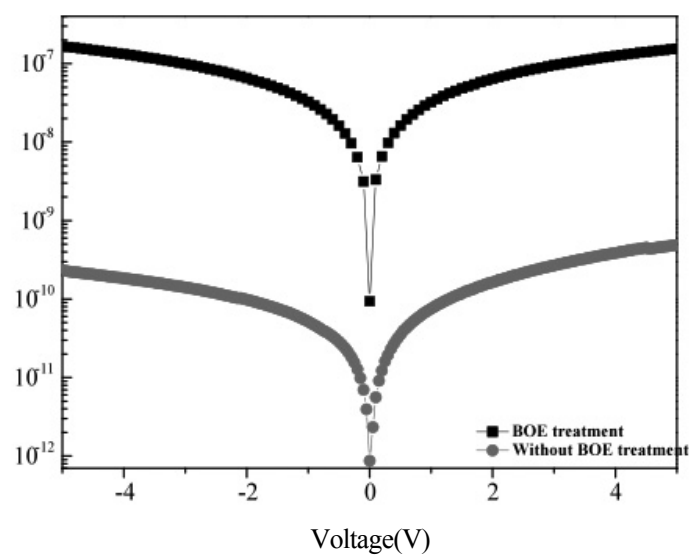

Fig. 4. Transport measurements (the I-V characteristics) of $\mathrm{STO} / \mathrm{KNO} / \mathrm{STO}$ heterostructure for a variety of oxygen pressures during the deposition of the STO and KNO thin films.

A proposed charge transfer from the KNO layer to the interface $(\mathrm{STO} / \mathrm{KNO})$ is associated with an increase in the internal electric field in the KNO layer, which originates from the polar discontinuity between KNO and STO. The changing characteristics of the surface influence the internal electric field. Recent experimental reports have revealed the importance of the relationship between the interface and the surface charge states $[15,16]$. It is promising that the $2 \mathrm{DEG}$ conducting properties are able to be controlled by the surface adsorbates. Therefore, the conducting properties of this new heterostructure are particularly useful for use in polar molecule sensors.

\section{CONCLUSIONS}

The properties of a 2DEG in the novel STO/KNO heterointerface have been investigated. The $(\mathrm{KO})^{-} /\left(\mathrm{TiO}_{2}\right)^{0}$ heterointerface acting as a p-type layer to create a $2 \mathrm{DHG}$ is not observed to have conducting properties. The novel $(\mathrm{SrO})^{0} /\left(\mathrm{NbO}_{2}\right)^{+}$heterointerface acting as an n-type layer to create a $2 \mathrm{DEG}$ is observed to have conducting properties. The oxygen pressures are controlled during the deposition of the KNO and STO thin films in the novel KNO/STO heterostructure. As a result, the effect of oxygen vacancies was observed at the heterostructure. The current level of the 2DEG can easily be controlled by changing the oxygen pressure of the STO and KNO thin films during deposition. To investigate the influence of the polar discontinuity according to the polar catastrophe model, the STO/KNO heterostructure was grown on an STO substrate, without using the BOE treatment. The 2DEG is not formed in the $\mathrm{STO} / \mathrm{KNO} / \mathrm{STO}$ without BOE treatment. Therefore, to create a $2 \mathrm{DEG}$ in the $\mathrm{STO} / \mathrm{KNO}$ heterostructure, the combined effect of both the oxygen vacancy mechanism and the polar discontinuity mechanism should be considered. Furthermore, the conducting properties of the new heterostructure are particularly useful in sensors, twodimensional conductors, and other electronics applications.

\section{REFERENCES}

[1] Y. Tokura and H. Y. Hwang, "Condensed-matter physics: Complex oxides on fire", Nat. Mater., Vol. 7, p. 694, 2008.

[2] J. W. Reiner, F. J. Walker, and C. H. Ahn, “Atomically engineered oxide interfaces", Science, Vol. 323, p. 1018, 2009.

[3] A. Brinkman, M. Huijben, M. van Zalk, J. Huijben, and U. Zeitler, "Magnetic effects at the interface between non-magnetic oxides", Nat. Mater., Vol. 6, p. 493, 2007.

[4] N. Reyren, S. Thiel, A. D. Caviglia, L. Fitting Kourkoutis, G. Hammerl, C. Richter, C. W. Schneider, T. Kopp, A.-S. Rueitschi, D. Jaccard, M. Gabay, D. A. Muller, J. M. Triscone, and J. Mannhart, 
"Superconducting interfaces between insulating oxides", Science, Vol. 317, p. 1196, 2007.

[5] Z. S. Popovic', S. Satpathy, and R. M. Martin, “Origin of the two-dimensional electron gas carrier density at the $\mathrm{LaAlO}_{3}$ on $\mathrm{SrTiO}_{3}$ interface", Phys. Rev. Lett., Vol. 101, p. 256801, 2008.

[6] A. Ohtomo and H. Y. Hwang, "A high-mobility electron gas at the $\mathrm{LaAlO}_{3} / \mathrm{SrTiO}_{3}$ heterointerface", Nature, Vol. 427, p. 423, 2004.

[7] N. Nakagawa, H. Y. Hwang, and D. A. Muller, "Why some interface can not be sharp", Nat. Mater., Vol. 5, p. 204, 2006.

[8] C. Noguera, "Polar oxide surfaces", J. Phys.-Condes. Matter, Vol. 12, p. R367, 2000.

[9] P. W. Tasker, "The stability of ionic crystal surfaces", J. Phys. C: Solid State Phys, Vol. 12, p. 4977, 1979

[10] A. Kalabukhov, R. Gunnarsson, J. Börjesson, E. Olsson, T. Claeson, and D. Winkler, "Effect of oxygen vacancies in the $\mathrm{SrTiO}_{3}$ substrate on the electrical properties of the $\mathrm{LaAlO}_{3} / \mathrm{SrTiO}_{3}$ interface", Phys. Rev. B, Vol. 75, p. 121404, 2007.

[11] M. K. Niranjan, Y. Wang, S. S. Jaswal, and E. Y. Tsymbal, "Prediction of a switchable two-dimensional electron gas at ferroelectric oxide interfaces", Phys.
Rev. Lett., Vol. 103, p. 016804, 2009.

[12] Y. Wang, M. K. Niranjan, S. S. Jaswal, and E. Y. Tsymbal, "First-principles studies of a twodimensional electron gas at the interface in ferroelectric oxide heterostructures", Phys. Rev. B, Vol. 80, p. 165130, 2009.

[13] H. N. Lee, H. M. Christen, M. F. Chisholm, C. M. Rouleau, and D. H. Lowndes "Thermal stability of epitaxial $\mathrm{SrRuO}_{3}$ films as a function of oxygen pressure", Appl. Phys. Lett., Vol. 84, 4107, 1956.

[14] M. Hiratani, Y. Tarutani, T. Fukazawa, and M. Okamoto, "Growth of $\mathrm{SrTiO}_{3}$ thin films by pulsedlaser deposition", Thin Solid Films, Vol. 227, p. 100, 1993.

[15] C. Cen, S. Thiel, J. Mannhart, and J. Levy "Oxide nanoelectronics on demand", Science, Vol. 323, p. 1026, 2009.

[16] C. Cen, S. Thiel, G. Hammerl, C. W. Schneider, K. E. Andersen, C. S. Hellberg, J. Mannhart, and J. Levy "Nanoscale control of an interfacial metal-insulator transition at room temperature", Nat. Mater., Vol. 7, p. 298, 2008. 